\title{
Potential barrier mimicking frequent location measurements in quantum Zeno dynamics
}

\author{
Miguel A. Porras \\ Grupo de Sistemas Complejos, Universidad Politécnica de Madrid, Rios Rosas 21, 28003 Madrid, Spain \\ Isabel Gonzalo and Alfredo Luis \\ Departamento de Óptica, Facultad de Ciencias Físicas, Universidad Complutense, 28040 Madrid, Spain
}

(Received 9 January 2016; published 7 April 2016)

\begin{abstract}
We show that quantum Zeno dynamics can be mimicked by the isolated evolution of an unobserved system in an effective potential. Monitoring frequently whether a particle remains in a region of space leads to the same wave-packet dynamics as placing the region on top of a potential barrier and letting the particle evolve on its own, without external couplings. We focus on very frequent but not continuous observation so that the particle abandons the initial region with some finite probability. The height of the barrier relative to the surroundings for a high frequency $v$ of the observations being mimicked is found numerically to be $h v / 2$, where $h$ is Planck's constant.
\end{abstract}

DOI: 10.1103/PhysRevA.93.040101

\section{INTRODUCTION}

In the beginning, the Zeno effect was presented as a consequence of the state reduction associated with quantum measurement [1]. Later, it was discovered that the controversial state reduction plays no role, so it does not even matter whether the planned measurement is finally carried out or not; it is enough that it could be made [2]. The Zeno effect is a purely dynamical consequence of the alteration of the observed system caused by the changes that must be introduced in the system in order to make possible the observation of the intermediate stages of the evolution [3]. Moreover, the fact that disputable quantum features play no role is clearly illustrated by the numerous demonstrations of Zeno-like dynamics in classical physics [4,5]. The interest in the research in quantum Zeno dynamics is evidenced by several proposals (e.g., [6]) as well as recent experiments [7-9], with application to the control of quantum states and to quantum information processing.

We investigate here the Zeno dynamics of a quantum particle that is frequently observed to see whether it remains in the region of space where it is initially found. For sufficiently high, but not infinite, frequency of the measurements, we demonstrate that the dynamics of the wave packet within the observation region is the same as that obtained by placing the region on top of a potential barrier and letting the particle evolve on its own. That is, quantum Zeno dynamics can be mimicked by the isolated evolution of an unobserved system. The dynamics of the particle with energy over the barrier and mimicking the Zeno dynamics is seen to result from the well-known quantum reflections at the discontinuities of the barrier.

By means of numerical simulations we find that the height $V_{0}$ of the barrier that reproduces the quantum Zeno dynamics with observation frequency $v$ is given by the simple formula $V_{0} \simeq h v / 2$. This is interpreted to be a consequence of the energy-time uncertainty principle $\Delta t \Delta E \gtrsim \hbar$ for a characteristic evolution time dictated by the observation period $1 / v$ being mimicked by an unobserved particle that can be located within or outside the barrier. Another key feature is that this result holds for other than perfect observation, i.e., in conditions of very frequent but not continuous observation, so that the particle abandons the initial region with some finite probability. The limit of continuous observation previously studied in [10] is seen to emerge naturally from our analysis in the strict limit $v=\infty$.

\section{ZENO DYNAMICS OF A QUANTUM PARTICLE}

We consider, for simplicity, a quantum particle of mass $m$ in a one-dimensional space $x$. The particle is prepared to be at a certain time $t=0$ in an interval $\Omega=\left[a_{1}, a_{2}\right]$, so that

$$
\int_{\Omega}|\psi(x, 0)|^{2} d x=1 .
$$

The wave function of the particle evolves according to the time-dependent Schrödinger equation

$$
i \hbar \partial_{t} \psi=-\frac{\hbar^{2}}{2 m} \partial_{x x} \psi+V \psi,
$$

but with period $T$ or frequency $v=1 / T$, the evolution is interrupted in order to observe whether the particle remains in the interval $\Omega$ or not. These observations are assumed to be instantaneous. If the particle is found in $\Omega$ in the first measurement at time $T$, the evolved wave function is set to zero out of $\Omega$, and its norm after projection in $\Omega$ gives the probability to find the particle in $\Omega$ at time $t=T$. The same applies to successive observations at times $t=2 T, 3 T, \ldots$. Formally, the wave function after the $n$th observation is given by

$$
\psi(x, n T)=\Pi e^{-i \frac{H T}{\hbar}} \stackrel{(n)}{.} . e^{-i \frac{H T}{\hbar}} \psi(x, 0),
$$

where $\Pi=1$ if $x \in \Omega$ and 0 otherwise, $H=p^{2} / 2 m+V$ is the Hamiltonian, and the norm of the wave function

$$
P(n T)=\int_{\Omega}|\psi(x, n T)|^{2} d x
$$

gives the probability to find the particle in $\Omega$ in all $n$ measurements. This is an example of quantum Zeno dynamics [10], characterized by a unitary evolution dictated by the Hamiltonian $H$, alternated by projections $\Pi$ on a certain subspace. The quantum Zeno dynamics generalizes 
the simpler quantum Zeno effect in which the observation concerns whether the particle remains in the initial state $[1,11]$. We point out that the above description is equivalent to that in which the wave function is not only projected but also normalized to unity in each observation:

$$
\frac{\psi(x, n T)}{\sqrt{P^{(n)} \cdots P^{(1)}}}=\frac{\Pi}{\sqrt{P^{(n)}}} e^{-i \frac{H T}{\hbar}} \stackrel{(n)}{.} \frac{\Pi}{\sqrt{P^{(1)}}} e^{-i \frac{H T}{\hbar}} \psi(x, 0),
$$

where $P^{(i)}$ gives the probability of finding the particle in $\Omega$ in the $i$ th measurement after having found the particle in $\Omega$ in the $(i-1)$ th measurement. The probability of finding the particle in $\Omega$ in all $n$ measurements is the product $P^{(n)} \ldots P^{(1)}$ of the individual probabilities. Since the norm of the left-hand side of Eq. (5) is unity, it follows that this product is given by $P(n T)$ in Eq. (4).

Let us first focus on the case of a free particle $(V=0)$. Figure 1 refers to the quantum Zeno dynamics of particles prepared in $\Omega=[-a, a]$ at rest [Fig. 1(a)] and moving at some speed [Fig. 1(b)], evolving freely but frequently observed to see whether they remain in $\Omega$. In the numerical simulations in this Rapid Communication and their results, such as those in Fig. 1, we use the dimensionless coordinate $x^{\prime}=x / a$ and the dimensionless time $t^{\prime}=t / t_{c}$, where $t_{c}=m a^{2} / \hbar$ characterizes the spreading time associated with a wave packet whose size is of the order of $a$. The wave function of an unobserved, free particle would evolve significantly at times $t^{\prime}$ of the order of unity, and a "frequent" observation is regarded to be that with period $T^{\prime}=T / t_{c}$ much smaller than unity. In these dimensionless variables the Schrödinger equation in Eq. (2) reads $i \partial_{t^{\prime}} \psi=-(1 / 2) \partial_{x^{\prime} x^{\prime}} \psi+V^{\prime} \psi$, where the dimensionless potential is $V^{\prime}=\left(t_{c} / \hbar\right) V$ and dimensionless momenta and energies are $p^{\prime}=p a / \hbar$ and $E^{\prime}=\left(t_{c} / \hbar\right) E$, respectively. All numerical simulations in this Rapid Communication have been performed using a standard, symmetrized split-step Fourier method (see, for example, [12]), in which the error due to discretization in time diminishes as $d t^{\prime 3}$ for a time step $d t^{\prime}$. Dealing with truncated functions or discontinuous potentials, errors may nevertheless be large due to subsampling in $x^{\prime}$ and $t^{\prime}$ in the fast spatial and temporal variations. In all simulations we have minimized these errors by choosing a sufficiently
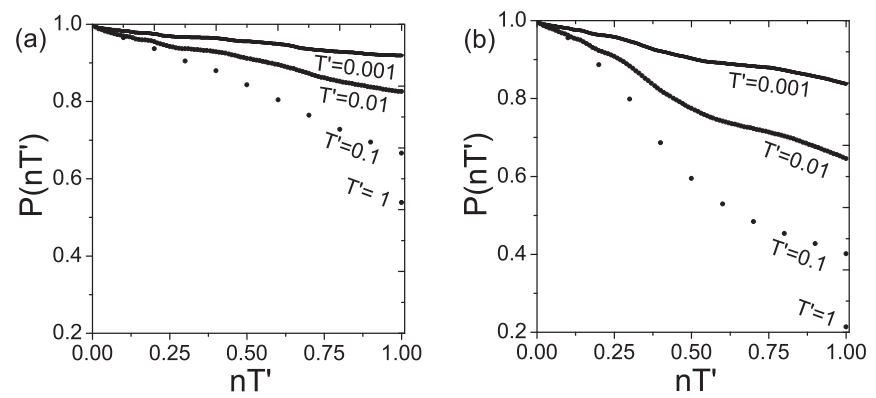

FIG. 1. Permanence probability $P\left(n T^{\prime}\right)$ in $[-1,1]$ in successive measurements at times $n T^{\prime}$ for a particle in the initial state $\psi\left(x^{\prime}, 0\right) \propto \operatorname{rect}\left(x^{\prime}\right) \exp \left(-x^{\prime 2}\right) \exp \left(i p^{\prime} x^{\prime}\right)\left[\operatorname{rect}\left(x^{\prime}\right)=1\right.$ if $x^{\prime} \in[-1,1]$ and 0 otherwise] for different frequencies $1 / T^{\prime}$ of the measurements. In (a) the particle is initially at rest $\left(p^{\prime}=0\right)$, and in (b) it is moving $\left(p^{\prime}=2\right)$. Upper curves seem like solid curves because the dots are very close. large number of points (e.g., changes cannot be detected at the scale of the figures when doubling the number of points).

The capacity of the frequent observation to confine the free particle, i.e., to inhibit wave-packet spreading and displacement, is clear from Figs. 1(a) and 1(b). The probability $P(n T)$ of finding the particle in its original location is seen to approach zero as time increases, but at any particular time this probability increases and approaches unity as the frequency $v$ of the measurements increases, as described previously in $[5,13]$.

\section{POTENTIAL BARRIER MIMICKING ZENO DYNAMICS}

In the limit of continuous measurements $(v=\infty)$, the Zeno dynamics within the observation region $\Omega$ was shown to be described by the Hamiltonian $H=p^{2} / 2 m+V_{\Omega}$, where $V_{\Omega}=$ 0 for $x \in \Omega$ and $V_{\Omega}=\infty$ otherwise; the particle thus remains with unit probability in $\Omega$ and behaves as an unobserved particle within an infinite potential well in $\Omega$ [10]. We may wonder if this fact can be extended below the limit of continuous measurements $v<\infty$, i.e., if the Zeno dynamics within the observation region $\Omega$, such as that in the examples in Fig. 1, can take place in an unobserved system subjected to a particular confining potential. If so, one expects, as in the limit $v=\infty$, this potential to be defined by the observation region $\Omega$ and to be independent of the particular initial state of the particle. At first glance, one is tempted to consider the finite potential well $V_{\Omega}=0$ for $x \in \Omega$ and $V_{\Omega}=V_{0}>0$ otherwise, with increasing height $V_{0}$ with the frequency of the measurements so as to recover the "hard-wall" potential in the limit of continuous observation. However, for an initial state with kinetic energy lower than $V_{0}$, the lingering probability in $\Omega$ of such a bounded state is constant in time (for an eigenstate) or oscillates about a constant value, while in the Zeno dynamics the lingering probability in $\Omega$ always approaches zero in time.

A lesser-known candidate also producing confinement is a finite potential barrier $V_{\Omega}=0$ in $\Omega$ and $V_{\Omega}=-V_{0}$ outside, with $V_{0}>0$, shown in Fig. 2 . A wave packet located initially within the barrier will certainly abandon it, but as the depth $V_{0}$ increases, the reflection coefficients of any plane-wave component $k=p / \hbar$ at the boundaries are known to increase, so the wave packet will spend more and more time in $\Omega$. For

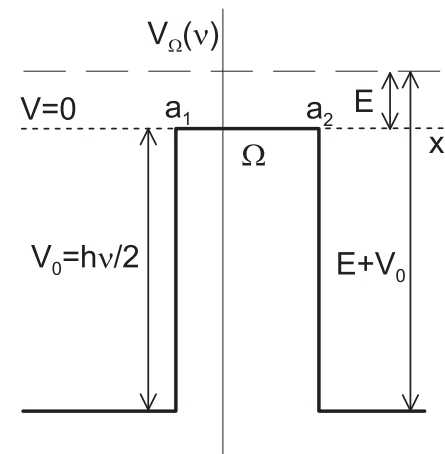

FIG. 2. The potential $V_{\Omega}(v)$ approaching in $\Omega$ the Zeno dynamics of a free particle subjected to frequent observation of permanence in $\Omega$. 

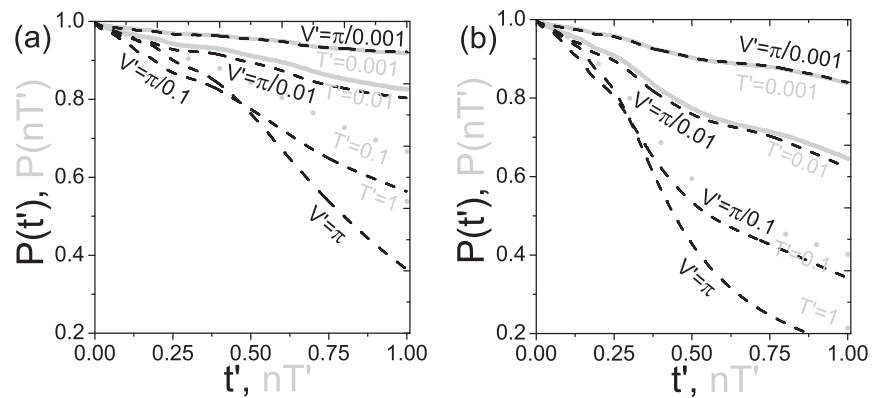

FIG. 3. Dashed curves: Permanence probability $P\left(t^{\prime}\right)$ in a barrier in $[-1,1]$ as a function of time for a particle of initial state $\psi\left(x^{\prime}, 0\right) \propto$ $\operatorname{rect}\left(x^{\prime}\right) \exp \left(-x^{\prime 2}\right) \exp \left(i p^{\prime} x^{\prime}\right)$ for different values $V_{0}^{\prime}=\pi / T^{\prime}$ of the barrier depth. In (a) $p^{\prime}=0$; in (b) $p^{\prime}=2$. Figure 1 is reproduced in gray for comparison.

example, Figs. 3(a) and 3(b) show the probability of finding the particle in $[-a, a]$ for the same initial states as in Figs. 1(a) and 1(b) but for the unobserved evolution under the action of potential barriers in $[-a, a]$ of increasing depths $V_{0}$ (the particular values are discussed below). As seen, increasing depths $V_{0}$ lead to increasing probabilities of finding the particle in the barrier at any given time. As in the Zeno dynamics, the probability tends, nevertheless, to zero at large time because a fraction of the wave function continuously escapes from the barrier, acquiring large kinetic energy and momentum towards $x= \pm \infty$.

We have found by numerical means that when the frequency of the measurements is finite but sufficiently high, the probabilities of finding the particle in $\Omega$ at any time in the Zeno dynamics and in the unobserved evolution under the action of a potential barrier of depth $V_{0}=h v / 2$ (or dimensionless depth $V_{0}^{\prime}=\pi / T^{\prime}$ ) tend to match. More precisely, the Zeno dynamics within $\Omega$ and the unobserved evolution within the potential barrier,

$$
V_{\Omega}(\nu)= \begin{cases}0 & \text { in } \Omega, \\ -h \nu / 2 & \text { out of } \Omega,\end{cases}
$$

approach one another asymptotically as $v \rightarrow \infty$. The potential in Eq. (6) is solely determined by the region and frequency of the observations being mimicked, irrespective of the particular initial state of the particle, as discussed below. In Figs. 3(a) and 3(b), for example, the probabilities in $[-a, a]$ in the Zeno dynamics with period $T$ and in the potential barrier of Eq. (6) with $v=1 / T$ are seen to approach one another as the period $T$ is increasingly shorter than the characteristic evolution time $t_{c}$ of the particle for both the initially still and moving particles. For the initially moving particle, Fig. 4 shows that it is not only the lingering probability but also the dynamics of the wave functions within $[-a, a]$ that becomes increasingly similar as $T / t_{c}$ approaches zero, as seen in Fig. 4(a) for $T<t_{c}$ and in Fig. 4(b) for $T \ll t_{c}$.

The validity of Eq. (6) asymptotically as $v \rightarrow \infty$ for different initial states is further supported by Fig. 5. For a particle in initial Gaussian-shaped states of different widths and velocities, the numerically evaluated depth $V_{0}$ of the potential barrier that yields the same lingering probability in $[-a, a]$ at a certain time $\left(t=t_{c}\right)$ as in the Zeno dynamics with frequency $v$ is depicted as this frequency increases. The
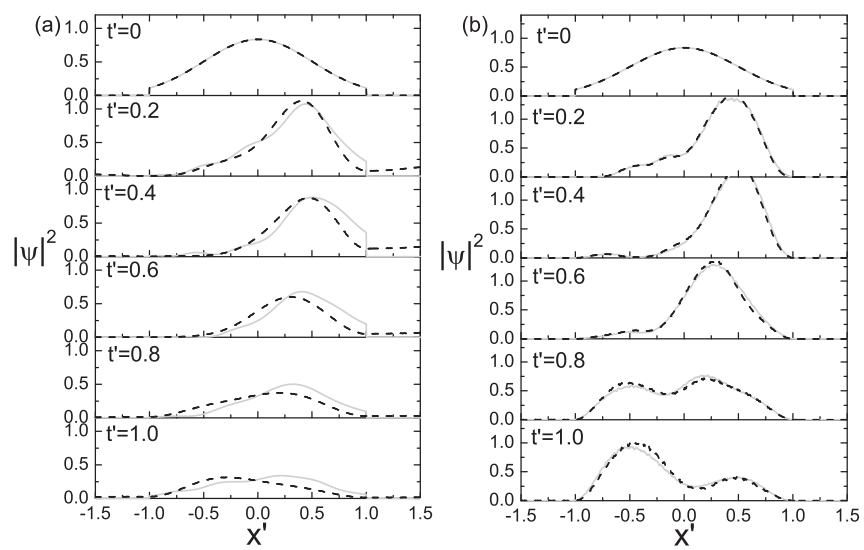

FIG. 4. Gray curves: Snapshots of the probability density in the Zeno dynamics with observation in $[-1,1]$ with (a) $T^{\prime}=0.1$ and (b) $T^{\prime}=0.001$ for a particle in the initial state $\psi\left(x^{\prime}, 0\right) \propto$ $\operatorname{rect}\left(x^{\prime}\right) \exp \left(-x^{\prime 2}\right) \exp \left(i p^{\prime} x^{\prime}\right), p^{\prime}=2$. Dashed curves: the same as the gray curves, but in the unobserved evolution with potential barriers in $[-1,1]$ of depth $V_{0}^{\prime}=\pi / T^{\prime}$.

numerical values of $V_{0}$ are seen to approach asymptotically the straight line $h v / 2$ irrespective of the initial state.

Other aspects are discussed in relation to Fig. 6, where the observation region is the unbounded interval $\Omega=(-\infty, a]$. For a particle launched towards positive $x$, the Zeno dynamics is seen to consist of the reflection of the particle towards negative $x$ with a probability that increases with the frequency of the measurements (e.g., $\simeq 0.8$ with $T=0.001 t_{c}$ ). In Fig. 6(a) the probability that the particle remains in $(-\infty, a]$ with potential barriers in $(-\infty, a]$ with depths higher than, equal to, and smaller than $V_{0}=h v / 2$ and the same initial state (cyan, black, and blue curves) is compared to the probability in the Zeno dynamics (gray curve). We note in Fig. 6(b) that the behavior of the wave functions in $(-\infty, a]$ is quite similar in the Zeno dynamics with frequent measurements and in

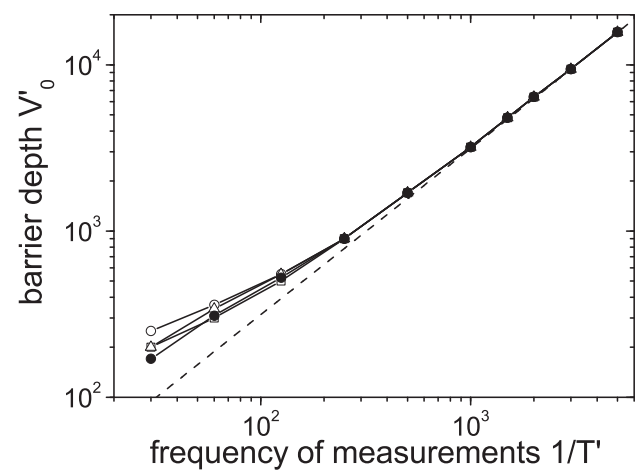

FIG. 5. For initial wave functions $\psi\left(x^{\prime}, 0\right) \propto$ $\operatorname{rect}\left(x^{\prime}\right) \exp \left(-x^{\prime 2} / s^{2}\right) \exp \left(i p^{\prime} x^{\prime}\right)$ with $s=1, p^{\prime}=0$ (open squares), $s=2, p^{\prime}=0 \quad$ (open circles), $s=1, p^{\prime}=1 \quad$ (triangles), and $s=1, p^{\prime}=2$ (closed circles), numerically evaluated depth $V_{0}^{\prime}$ of the potential barrier in $[-1,1]$ that gives the same lingering in probability in $[-1,1]$ at $t^{\prime}=1$ as in the Zeno dynamics with measurements in $[-1,1]$ for increasing values $1 / T^{\prime}$ of the frequency of the measurements. The dashed line is the dimensionless depth $V_{0}^{\prime}=\pi / T^{\prime}$ of the barrier in Eq. (6). 

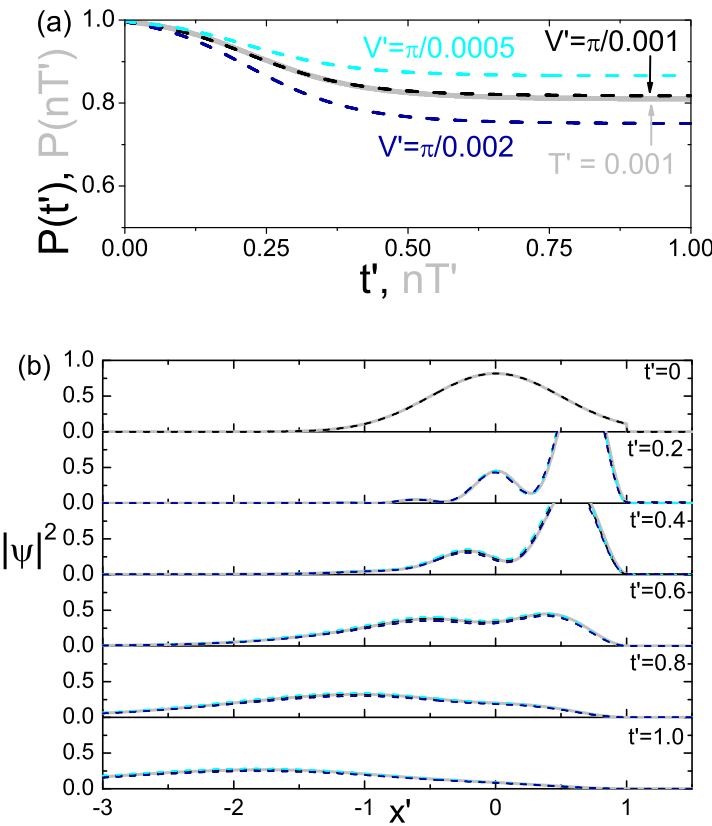

FIG. 6. (a) For a particle in the initial state $\psi\left(x^{\prime}, 0\right) \propto$ $\exp \left(-x^{\prime 2}\right) \exp \left(i p^{\prime} x^{\prime}\right)$ in $(-\infty, 1]$ and $\psi\left(x^{\prime}, 0\right)=0$ otherwise, with $p^{\prime}=4$, lingering probability $P\left(n T^{\prime}\right)$ in $(-\infty, 1]$ at the $n$th measurement at $n T^{\prime}$ with period $T^{\prime}=0.001$ (gray curve) and lingering probability $P\left(t^{\prime}\right)$ in $(-\infty, 1]$ in barriers in $(-\infty, 1]$ of different depths $V_{0}^{\prime}=\pi / 2 T^{\prime}$ (blue curve), $V_{0}^{\prime}=\pi / T^{\prime}$ (black curve), and $V_{0}^{\prime}=2 \pi / T^{\prime}$ (cyan curve). (b) Snapshots of the probability density for the same particle in the Zeno dynamics with $T^{\prime}=0.001$ (gray curves) and for the unobserved evolution with potential barriers in $(-\infty, 1]$ of depths $V_{0}^{\prime}=\pi / 2 T^{\prime}$ (blue curve), $V_{0}=\pi / T^{\prime}$ (black curve), and $V_{0}=2 \pi / T^{\prime}$ (cyan curve).

the unobserved evolution for all three deep potential barriers (the gray and colored curves are barely distinguishable) since all of them approximate, to a higher or lower degree, the Zeno dynamics in the limit of continuous measurements. The relevant point here is that it is with a barrier of depth $V_{0}=h v / 2$ that the norm of the wave function in $(-\infty, a]$ for the Zeno dynamics tends also to fit, with this fitting being more accurate as the frequency of the measurements increases.

Although it may seem contradictory at first, it is not hard to understand why the infinite potential well describes continuous observation as the limit of increasingly deep potential barriers for increasing frequency of the observation. For a wave packet located within the barrier $V_{\Omega}(v)$, the reflection coefficient of any plane-wave component $k$ at the boundaries can be evaluated to be [14] $R=\left|k-k_{\Omega}\right|^{2} /\left|k+k_{\Omega}\right|^{2}$, where $k_{\Omega}=$ $\sqrt{2 m E} / \hbar, k=\sqrt{2 m\left(E+V_{0}\right)} / \hbar$ and $E$ is the kinetic energy in $\Omega$ (see Fig. 2). The reflection coefficient $R$ then approaches unity as $V_{0}=h v / 2 \rightarrow \infty$. In this limit, the particle located initially in $\Omega$ then finds hard walls at its boundaries, as in the infinitely high potential well that describes the Zeno dynamics under continuous observation [10].

The above results for a free particle admit a generalization, also corroborated by numerical simulations, to the Zeno dynamics of a particle subjected to a potential $V$ of any particular shape, provided that it is continuous. The equivalent
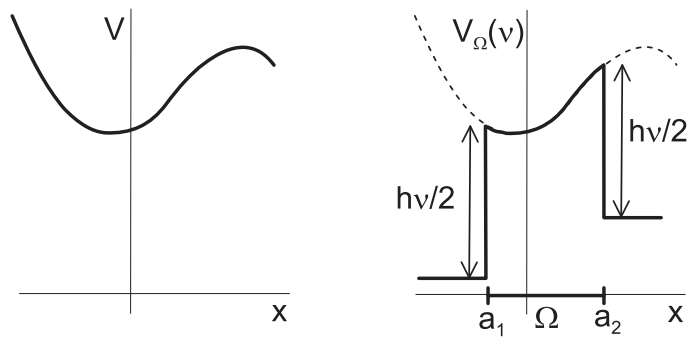

FIG. 7. A potential and the potential $V_{\Omega}(v)$ approaching in $\Omega$ the Zeno dynamics of a particle subjected to the potential $V$ and frequent observations of permanence in $\Omega$.

potential that mimics the Zeno dynamics is found to be

$$
V_{\Omega}(\nu)= \begin{cases}V & \text { in } \Omega, \\ V(\partial \Omega)-h \nu / 2 & \text { out of } \Omega,\end{cases}
$$

where $V(\partial \Omega)$ represents the two values of the potential at each of the boundaries of $\Omega=\left[a_{1}, a_{2}\right]$. The potential $V_{\Omega}(v)$ in Eq. (7) is depicted in Fig. 7 for clarity. The "jumps" on both boundaries are equal to $h v / 2$.
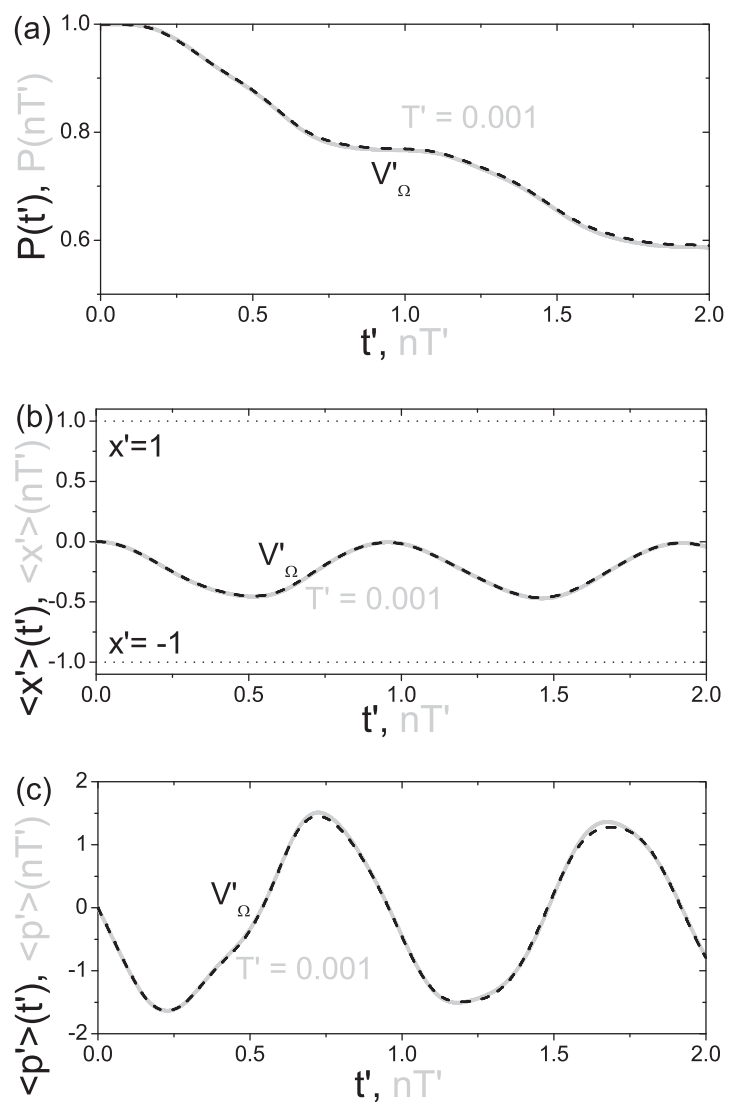

FIG. 8. For a particle in the initial state $\psi\left(x^{\prime}, 0\right) \propto$ $\operatorname{rect}\left(x^{\prime}\right) \exp \left[-\left(x^{\prime} / 0.5\right)^{2}\right]$ subjected to the potential $V^{\prime}=10 x^{\prime}$, (a) probability of permanence in $[-1,1]$, (b) expected value of position, and (c) expected value of momentum in $[-1,1]$, when the particle is observed to be in $[-1,1]$ with period $T^{\prime}=0.001$ (gray curves) and when it is not observed but subjected to the potential $V_{\Omega}^{\prime}=$ $10 x^{\prime}$ in $[-1,1], V_{\Omega}^{\prime}=10-\pi / T^{\prime}$ in $(1, \infty)$, and $V_{\Omega}^{\prime}=-10-\pi / T^{\prime}$ in $(-\infty,-1)$ (dashed curves). 
As an example of the validity of Eq. (7), we consider a particle initially at rest in $[-a, a]$, subjected to a constant force $-F<0$ towards negative $x$, i.e., to the potential $V=F x$, and frequently observed to see whether it remains in $[-a, a]$. The gray curves in Fig. 8 depict the probability of finding the particle and the expected values of position $x$ and momentum $p$ as functions of the measurement time $n T$. Although the particle initially accelerates towards negative $x$, the frequent observation makes it to bounce repeatedly at the boundary $x=$ $-a$ with a probability that decreases with time. The dashed curves in Fig. 8 correspond to the probability and expected values of position and momentum in the region $[-a, a]$ for the unobserved evolution under the potential in Eq. (7), which reads in this case $V_{\Omega}(v)=F x$ in $[-a, a], V_{\Omega}(v)=F a-h v / 2$ in $(a, \infty)$, and $V_{\Omega}(v)=-F a-h v / 2$ in $(-\infty,-a)$. Since the frequency is high, this potential describes accurately the Zeno dynamics.

\section{DISCUSSION AND CONCLUSIONS}

It is not difficult to understand the proportionality to $h v$ of the equivalent potential barrier as a requirement of the energytime uncertainty principle $\Delta t \Delta E \gtrsim \hbar$. For a particle subjected to a potential $V, \Delta E$ is the uncertainty in the energy, and $\Delta t$ is a characteristic evolution time, such as $t_{c}$. Measurements with period $T \ll t_{c}$ impose the much faster characteristic time $\Delta t=T$ for the Zeno dynamics. These measurements involve an uncontrollable exchange of energy between the particle and the measurement apparatus [15-17] with an uncertainty $\Delta E$ such that $T \Delta E \gtrsim \hbar$. For the potential barrier, on the other hand, simulating the Zeno dynamics requires $\Delta t=T$, and the uncertainty in the energy $\Delta E$ is substantially related to whether the particle is inside or outside the barrier and therefore of the order of $V_{0}$. The uncertainty principle then imposes $V_{0} \gtrsim$ $h \nu / 2 \pi$ for the height of the barrier, which is satisfied for all frequencies by the value $V_{0}=h v / 2$.

In conclusion, we have found that the Zeno dynamics of a quantum particle evolving according to a Hamiltonian $H=p^{2} / 2 m+V$ and frequently observed to see whether it remains in a region of space $\Omega$ is equivalent asymptotically at large observation frequency to the dynamics within $\Omega$ of the unobserved particle evolving according to the Hamiltonian $H_{\Omega}=H+V_{\Omega}(v)$, with $V_{\Omega}(v)$ given by Eq. (7). This describes how the hard-wall Dirichlet conditions at the boundary of the observation region in the limit of continuous observation are reached below this limit. A consequence of this investigation is that the frequent measurements can be replaced by a potential energy. This makes the observed particle act as if it had an extra energy $V_{0}=h v / 2$ while it remains in the observation region and an energy uncertainty of the order of $h v / 2$ related to its location inside or outside the region. In the Zeno scheme mimicked by the potential barrier, the energy exchange between the particle and the measurement apparatus [15-17] can then be said to be of the order of $h v / 2$.

\section{ACKNOWLEDGMENTS}

We acknowledge support from projects of the Spanish Ministerio de Economía y Competitividad No. MTM201239101-C02-01 (M.A.P.), No. MTM2015-63914-P (M.A.P.), No. FIS2013-41709-P (M.A.P. and I.G.), and No. FIS201235583 (A.L.) and from the Comunidad Autónoma de Madrid research consortium QUITEMAD+ Grant No. S2013/ICE2801 (A.L.). M.A.P. acknowledges helpful discussions with J. Usera.
[1] B. Misra and E. C. G. Sudarshan, J. Math. Phys. 18, 756 (1977).

[2] D. Home and M. A. B. Whitaker, Ann. Phys. (N.Y.) 258, 237 (1997); J. Peise, B. Lücke, L. Pezzé, F. Deuretzbacher, W. Ertmer, J. Arlt, A. Smerzi, L. Santos, and C. Klempt, Nat. Commun. 6, 6811 (2015).

[3] T. P. Altenmüller and A. Schenzle, Phys. Rev. A 49, 2016 (1994); S. Pascazio and M. Namiki, ibid. 50, 4582 (1994); H. Nakazato, M. Namiki, S. Pascazio, and H. Rauch, Phys. Lett. A 199, 27 (1995); A. Venugopalan and R. Ghosh, ibid. 204, 11 (1995); A. Luis and J. Peřina, Phys. Rev. Lett. 76, 4340 (1996).

[4] M. Kitano, Opt. Commun. 141, 39 (1997); A. Peres, Am. J. Phys. 48, 931 (1980); K. Yamane, M. Ito, and M. Kitano, ibid. 192, 299 (2001); P. Kwiat, H. Weinfurter, T. Herzog, A. Zeilinger, and M. Kasevich, Ann. N.Y. Acad. Sci. 755, 383 (1995); S. Longhi, Phys. Rev. Lett. 97, 110402 (2006).

[5] M. A. Porras, A. Luis, I. Gonzalo, and A. S. Sanz, Phys. Rev. A 84, 052109 (2011); M. A. Porras, A. Luis, and I. Gonzalo, ibid. 88, 052101 (2013).

[6] J. M. Raimond, C. Sayrin, S. Gleyzes, I. Dotsenko, M. Brune, S. Haroche, P. Facchi, and S. Pascazio, Phys. Rev. Lett. 105, 213601 (2010); J. M. Raimond, P. Facchi, B. Peaudecerf, S.
Pascazio, C. Sayrin, I. Dotsenko, S. Gleyzes, M. Brune, and S. Haroche, Phys. Rev. A 86, 032120 (2012); X. Q. Shao, L. Chen, S. Zhang, and K.-H. Yeon, J. Phys. B 42, 165507 (2009); Z. C. Shi, Y. Xia, H. Z. Wu, and J. Song, Eur. Phys. J. D 66, 127 (2012).

[7] A. Signoles, A. Facon, D. Grosso, I. Dotsenko, S. Haroche, J.-M. Raimond, M. Brune, and S. Gleyzes, Nat. Phys. 10, 715 (2014).

[8] F. Schäfer, I. Herrera, S. Cherukattil, C. Lovecchio, F. S. Cataliotti, F. Caruso, and A. Smerzi, Nat. Commun. 5, 3194 (2014).

[9] G. Barontini, L. Hohmann, F. Haas, J. Estéve, and J. Reichel, Science 349, 1317 (2015).

[10] P. Facchi, S. Pascazio, A. Scardicchio, and L. S. Schulman, Phys. Rev A 65, 012108 (2001); P. Facchi and S. Pascazio, Phys. Rev. Lett. 89, 080401 (2002); J. Phys. A 41, 493001 (2008).

[11] L. A. Khalfin, Zh. Eksp. Teor. Fiz. 33, 1371 (1957) [Sov. Phys. JETP 6, 1053 (1958)]; C. Teuscher and D. Hofstadter, Alan Turing: Life and Legacy of a Great Thinker (Springer, Berlin, 2004), p. 54; A. Degasperis, L. Fonda, and G. C. Ghirardi, Nuovo Cimento A 21, 471 (1974). 
[12] G. P. Agrawal, Nonlinear Fiber Optics (Academic, San Diego, 2001).

[13] M. A. Porras, A. Luis, and I. Gonzalo, Phys. Rev. A 90, 062131 (2014).

[14] Supposing $A e^{i k_{\Omega} x}+B e^{-i k_{\Omega} x}$ in $\Omega, C e^{-i k x}$ to the left, and $D e^{i k x}$ to the right, a standard procedure [18] yields this result.

[15] A. Messiah, Mechanique Quantique (Dunod, Paris, 1964), Vol. 1.
[16] M. H. Partovi and R. Blankenbecler, Phys. Rev. Lett. 57, 2887 (1986).

[17] P. Busch, in Time in Quantum Mechanics, edited by J. G. Muga, R. Sals, and I. L. Egusquiza (Springer, Berlin, 2008), Vol. 1, Chap. 3, p. 81.

[18] C. Cohen Tannoudji, B. Diu, and F. Laloë, Quantum Mechanics (Wiley, New York, 1977), Vol. 1, p. 72. 